\title{
The Responses of HIF-1 $\alpha$ and HSP70 to Interval Training with Various Intensities in Indonesian Junior Sprinters
}

\author{
Eddy Purnomo ${ }^{1, *}$ Mohamad Sadikin $^{2}$ \\ ${ }^{1}$ Faculty of Sport Sciences, Universitas Negeri Yogyakarta, Yogyakarta, Indonesia \\ ${ }^{2}$ Faculty of Medicine, Universitas Indonesia, Jakarta, Indonesia \\ *Corresponding author. Email: eddy_purnomo@uny.ac.id
}

\begin{abstract}
Interval training (IT) is a method of exercise to improve running ability in predetermined distance and time. This study aims to describe physiological responses (hypoxia-inducible factor- $1 \alpha$ (HIF-1 $\alpha$ ) and heat shock proteins 70 (HSP70)) to several IT in male Indonesian junior sprinters. In this experimental study 17 male junior sprinters were allocated into 3 groups based on exercise intensity: low, moderate and high. Measured were height, body weight, $100 \mathrm{~m}$ running speed and biochemical parameters (HIF-1 $\alpha$ and HSP70), before IT, during IT, and after a 30-minutes break following IT. Data analyses were performed using Kruskal-Wallis and ANOVA tests. HIF-1 $\alpha$ concentration increased in high intensity group after IT compared to the condition during IT was performed, while HSP70 concentration mostly increased in moderate and high intensity groups. HSP70 concentrations were lower in all groups, after the 30-minute break, but did not recover to its concentration before IT was performed. HIF-1 $\alpha$ and HSP70 production depended on training intensity. Their concentrations did not reach baseline concentrations after 30-minute break. It is suggested that more than 30 minutes of rest are needed to recover after a very intense training session.
\end{abstract}

Keywords: Interval training, $H I F-1 \alpha, H S P 70$

\section{INTRODUCTION}

Systematic repetitive training, with an increased load everyday according to the target, is needed to improve athletes' performances and achievements. Its components consist of intensity, volume, duration, rhythm, and frequency. The target is usually set by adjusting the intensity with athletes' conditions. Intensity is defined as the quality of the exercise and can be determined by either distance/volume of exercise completed in each session or the running speed in predetermined time. Low-intensity training is an aerobic training while high-intensity training is typically anaerobic and may cause injuries or health problems if performed without sufficient recovery periods [1-3].

One of current methods developed in athletics is interval training (IT). The elements of IT are distance, time, resting period between exercises, number of repetitions and training intensity. This type of trainings comprises sequences of various intensities of trainings (low to high) and a resting period in between each training. IT applies generally to the athletes who want to improve their performance, particularly sprinters who want to improve their running speed in predetermined distance and time. The initial physiological response to exercise is an increased blood flow to muscle tissue. This increment lasts up to 25 times during training and is needed for muscle contraction.

The energy is produced aerobically in adequate oxygen supply milieu. However, repetitive or continuous high-intensity training, particularly in short period, will result in reduced oxygen concentration in muscle tissue which leads to anaerobic metabolism [4-6]. Anaerobic energy turnover generates lactic acid, which in turn causes fatigue declining the muscle's ability to contract $[1,4]$.

Birch and McLaren [7] found that high intensity of IT causes relative hypoxia depending on physiological muscle characteristics and the amount of energy supply required during training. Sustained hypoxia threatens the survival of cells, tissues, and organs [8]. Hence, the body has various mechanisms to adapt to hypoxia, such as stabilizing hypoxia inducible factor- $1 \alpha(\mathrm{HIF}-1 \alpha)[9,10]$ and to maintain homeostasis by inducing the production of heat shock protein (HSP) $[11,12]$. From the HSP subfamilies (due to molecular weight HSP27, HSP60, HSP70 and HSP90), especially HSP70 is easily increased in skeletal muscle by physical training [13].

To adjust IT to the conditions of Indonesian athletes, we here investigate HIF-1 $\alpha$, HSP70 under different exercise training intensities. 


\section{METHODS}

\section{A. Participants}

Seventeen male sprinters (mean \pm standard deviation, age: $15.6 \pm 0.7$ years; height: $168.1 \pm 3.3 \mathrm{~cm}$; weight: $54.4 \pm 3.3$ $\mathrm{kg}$ ) and fifteen male non-sprinters (mean \pm standard deviation, age: $15.4 \pm 0.6$ years; height: $161.1 \pm 3.3 \mathrm{~cm}$; weight: $51.5 \pm 2.9 \mathrm{~kg}$ ) participated in the study. The sprinters were joining Nasional Training Center in Indonesia but the non-sprinters were not joining it. Each group consisted of sprint athletes and non sprint-athletes was divided into 3 sub-groups randomly. There are high-intensity (90\%-100\%) group, moderate-intensity $(70 \%-80 \%)$ group, and lowintensity $(50 \%-60 \%)$ group.

\section{B. Experimental Design}

This study is an experimental study aiming to measure the response of a $10 \times 100$ meter interval running. Blood sampling, height, and body weight measurement were carried out at Senayan Madya Stadium. Blood analysis for HIF-1 $\alpha$ and HSP70 was done at Biochemistry Laboratory Faculty of Medicine, University of Indonesia. To measure body physiological response towards interval training (IT), blood sampling was carried out 3 times: before training, during training, and after a 30-minute break following training. The measurement was done by 3 steps. The first step: before training, anthropometry, first blood sampling and the ability to run $100 \mathrm{~m}$ were conducted. The results were used to classify subjects into the sub-groups. The second step, all subjects underwent $10 \times 100 \mathrm{~m}$ interval running with resting periods in between. Pulse rate should be in the range of 110-120 beats per minute (bpm). Soon after all subjects finished the running, blood sample was drawn. The third step, right after a 30 -minute resting period following IT, the third blood sample was taken. Blood samples were analyzed for HIF-1 $\alpha$ using enzyme-linked immunosorbent assay (ELISA) sandwich method (SurveyorTM IC, Human/Mouse HIF-1 $\alpha$ immunoassay, catalog number SUV1935), while HSP70 measurement used ELISA sandwich method (surveyorTM IC, human/mouse/rat total HSP70/HSPA1A immunoassay, catalog number SUV 1663).

C. Statistical Analyses

Data analyses were performed using R HIF-1 $\alpha$ and HSP70 values were statistically analyzed using $\mathrm{R}$ version 3.5.1. Mean differences between sprinters and non-sprinters' physical characteristics were assessed using Student's t-test. A statistical significant was obtained by setting up the alpha level at $<0.05$. A two-way analysis of variance (ANOVA) was performed to test the effect of the three intensity (low, moderate, high) and sprinters/non-sprinters on running speed. Normality and homogeneity of variances were checked using Shapiro Wilk test and Levene's test, respectively. In conjunction with the significance an ANOVA test, pos hoc comparisons using Tukey's honestly significance difference (HSD) were carried out. A KruskallWallis test was performed to test the difference in HIF-1 $\alpha$ concentration of sprinters between the intensities since the normality was not satisfied. A one-way ANOVA test was carried out to test the difference in HSP70 levels of sprinters between the intensities.

\section{Ethics}

The protocol was approved by the faculty of medicine ethical committee at the University of Indonesia (Number: 130/PT02.FK/ETIK/ 2011) and all subjects agreed to participate in this study by signing an informed consent.

\section{RESULTS}

Mean of age and body weight were about the same between sprinter and non-sprinter $(\mathrm{p}=0.889$ and $\mathrm{p}=0.432)$. Mean of body height for sprinter was higher than nonsprinter $(p=0.026)$. Its contrast with this result, the running speed $100 \mathrm{~m}$ for sprinter was faster than non-sprinter $(p=0.025)$. Moreover, based on each intensity, athletes' running speed was also faster than non- sprinter, as depicted in Fig. 1.

I.

Table 1. Physical characteristics of the subjects

\begin{tabular}{llll}
\hline Subjects Data & Sprinter $(\mathrm{n}=17)$ & $\begin{array}{l}\text { Non-sprinter } \\
(\mathrm{n}=15)\end{array}$ & $\mathrm{p}$ \\
\hline Age (year) & $15.6 \pm 0.7$ & $15.4 \pm 0.6$ & 0.889 \\
Body height $(\mathrm{cm})$ & $168.1 \pm 3.3$ & $161.1 \pm 3.3$ & $0.026^{*}$ \\
Body weight $(\mathrm{kg})$ & $54.4 \pm 3.3$ & $51.5 \pm 2.9$ & 0.432 \\
$\begin{array}{l}\text { Running speed 100 } \\
\mathrm{m}(\mathrm{s})\end{array}$ & $11.5 \pm 0.1$ & $13.5 \pm 0.1$ & $0.025^{*}$ \\
\hline
\end{tabular}

Data are presented as mean $\pm \mathrm{SD} ;{ }^{*} \mathrm{p}<0.05$.

In order to test the hypothesis that intensity level (low, moderate, high) and sprinter and non-sprinter had an interaction effect on running speed, a two-way ANOVA was performed. Prior to conducting the ANOVA, the assumption of normality for each 6 level combinations was examined using a Shapiro-Wilk test and found to be satisfied as each combination of the groups' distribution was associated with p-value greater than 0.05 . Furthermore, the assumption of homogeneity variances was tested and satisfied based on Levene's $F$ test, $F(5,26)=1.766$ and $p=0.155$. A two-way ANOVA yielded a main effect for the intensity, $F(2,26)=5.966, p=0.007$, indicating that there was a significant difference in mean running speed between the intensities. Pos hoc comparisons using the Tukey was carried out. There was a significant difference between low and moderate intensities $(\mathrm{p}=0.006)$ with people on moderate intensity run on average $0.37 \mathrm{~s}$ faster than those on low intensity, while the low-hard intensities and moderate-hard intensities showed the same lack of effect $(p=0.083$ and $\mathrm{p}=0.462$, respectively). The main effect of sprinter /nonsprinter yielded an $\mathrm{F}$ ratio of $\mathrm{F}(1,26)=466.536$ and $\mathrm{p}<0.001$. A Tukey's HSD further indicated that the mean running speed was significantly faster for sprinter $(M=11.465$, $\mathrm{SD}=0.150)$ than for non- sprinter $(\mathrm{M}=13.373, \mathrm{SD}=0.373)$. The interaction effect was non-significant, $\mathrm{F}(2,26)=2.328$, $\mathrm{p}=0.117$. The Tukey's HSD for interaction effect demonstrated the average running speed for sprinter on either low, moderate, or hard intensity was significantly faster than those non-sprinter (all p-values $<0.001$ ). 


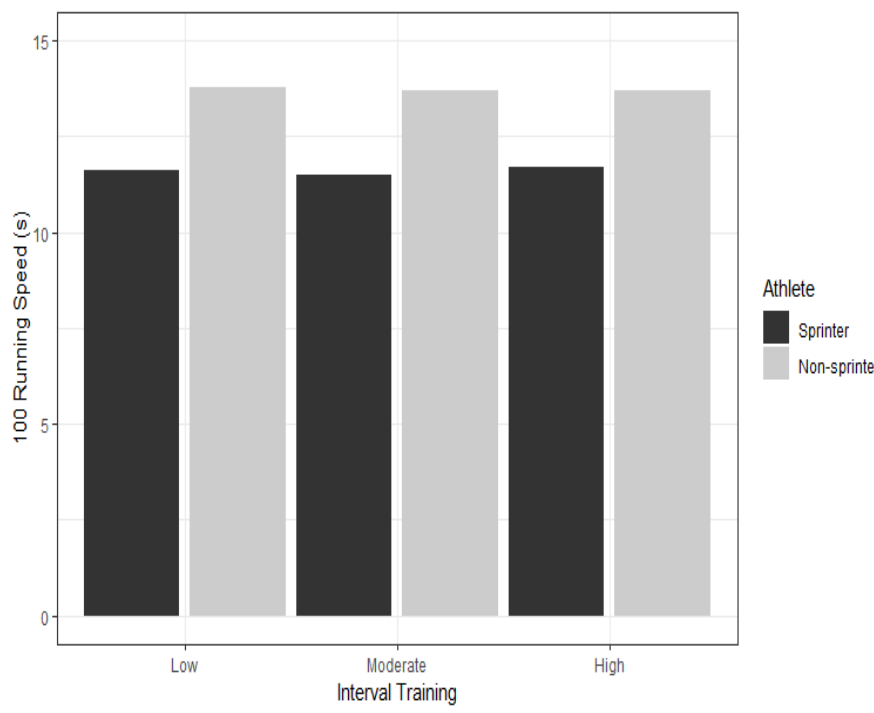

FIG. 1. Mean value of subjects' running speed in each intensity group

\section{A. Concentration of HIF-1 $\alpha$ and training intensity}

HIF-1 $\alpha$ concentration was measured before IT, during IT, and after a 30-minute break in all intensity groups. At baseline, a Kruskal-Wallis test showed that there was no statistically difference in HIF- $1 \alpha$ concentration of sprinters between the different intensities before IT $\left(\chi_{(2)}^{2}=0.666\right.$ and $\left.\mathrm{p}=0.717\right)$, during IT $\left(\chi_{(2)}^{2}=0.372\right.$ and $\mathrm{p}=0.830)$, and after 30-minute break $\left(\chi_{(2)}^{2}=0.058\right.$ and $\mathrm{p}=0.972$ ). The Kruskal-Wallis test was conducted on each group since the normality assumption on ANOVA was not satisfied.

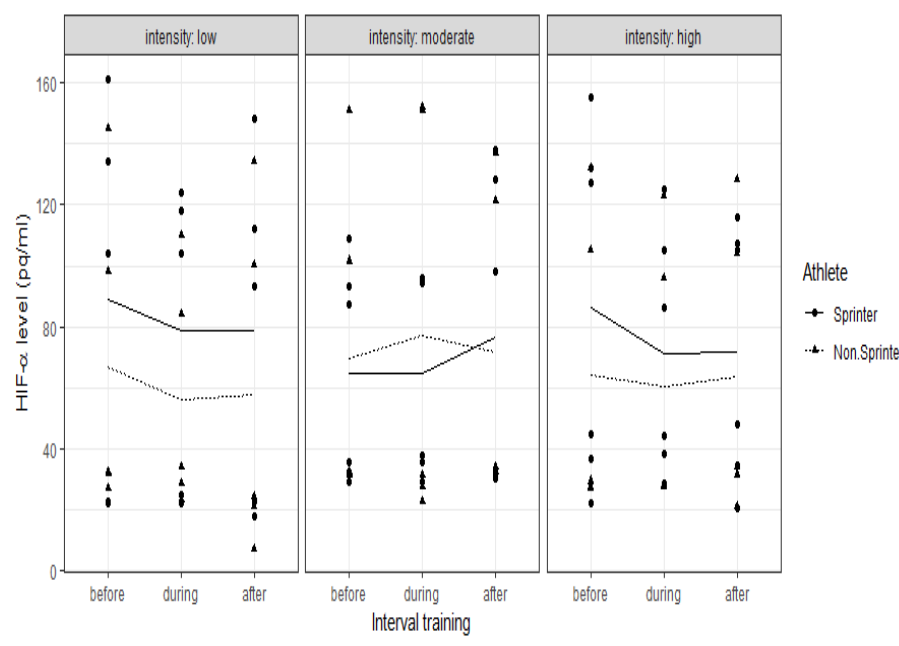

FIG. 2. HIF-1 $\alpha$ level before IT, during IF, and after recovery period based on training intensity for sprinter and non-sprinter athletes

Fig. 2 shows the observed and mean HIF- $\alpha$ levels given by participants in each of the nine conditions. When participants had low or high intensity for both sprinters and non-sprinters, the HIF- $\alpha$ levels were higher before IT than when participants were during IT or after break 30 minutes. The trend was changed in the moderate intensity for sprinters, while there was up and down trend for nonsprinters. Based on Table 2, no significant difference of
HIF- $1 \alpha$ concentration or percentage rise was found during and after IT in high-intensity $(\mathrm{p}=0.905)$, moderate-intensity $(p=0.225)$ nor low-intensity $(p=0.955)$ groups. However, there was elevation difference of HIF-1 $\alpha$ level among exercise intensity groups during and after IT: high-intensity (by $0.744 \%$ ) and low-intensity (by $0.475 \%$ ) groups $v s$ moderate-intensity group by $18.086 \%$.

Table 2. Sprinters' HIF-1 $\alpha$ level during and after IT in all intensity groups

\begin{tabular}{|c|c|c|c|c|c|}
\hline \multirow{2}{*}{$\begin{array}{l}\text { Training } \\
\text { Intensity }\end{array}$} & \multicolumn{2}{|c|}{ HIF-1 $\alpha$ Level (pg/ml) } & \multirow{2}{*}{$\begin{array}{l}\text { Difference } \\
(\mathrm{pg} / \mathrm{ml})\end{array}$} & \multirow{2}{*}{$\begin{array}{c}\text { Percen } \\
\text { tage } \\
(\%)\end{array}$} & \multirow[t]{2}{*}{$\mathrm{p}$} \\
\hline & During IT & After IT & & & \\
\hline High & $71.395 \pm 39.655$ & $71.926 \pm 42.112$ & 0.531 & 0.744 & 0.905 \\
\hline Moderate & $64.822 \pm 33.633$ & $76.546 \pm 50.968$ & 11.724 & 18.086 & 0.225 \\
\hline Low & $78.525 \pm 50.833$ & $78.989 \pm 56.965$ & 0.373 & 0.475 & 0.955 \\
\hline
\end{tabular}

\section{B. Concentration of HSP70 and training intensity}

HSP70 concentrations before IT, during IT, and after a break in each group are presented in Fig. 3 presents the observed and the mean HSP70 levels given by participants in each of the nine conditions. The mean value of HSP70 concentration of sprinters increased during IT and after a break in high- and moderate-intensity groups. The HSP70 concentration in high-intensity group increased $45.2 \%$ during IT and $2 \%$ after 30 -minute break compared to its concentration before IT. Similarly, it increased in the moderate training group by $49.5 \%$ during IT and $42.2 \%$ after the break period compared to its concentration before IT. Meanwhile in low-intensity group, HSP70 concentration decreased $11.3 \%$ during IT and further down $54.5 \%$ after the break compared to its concentration before IT (Fig. 3). The HSP70 levels for sprinters were significantly higher than non-sprinters across intensity groups $(\mathrm{p}<0.0001)$.

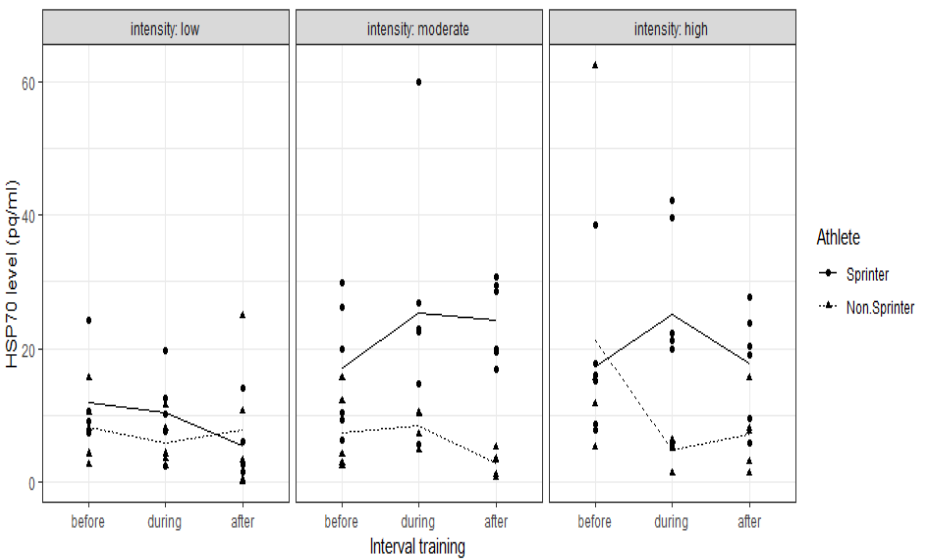

FIG. 3 HSP70 level before IT, during IF, and after recovery period based on training intensity for sprinter and nonsprinter athletes

A one-way ANOVA was conducted to compare the effectiveness of three intensities for sprinters on each IT. Normality checks using Shapiro Wilk's test and homogeneity of variances using Levene's test were carried out and the assumptions met. There was no significant difference in mean HSP70 levels of sprinters between the intensities before IT $(\mathrm{F}(2,14)=0.539, \mathrm{p}=0.595)$ and during IT $(\mathrm{F}(2,14)=1.922, \mathrm{p}=0.183)$. The significant difference in 
mean HSP70 levels of sprinter between the intensities was found after IT $(\mathrm{F}(2,14)=10.676, \mathrm{p}=0.002)$. Pos hoc comparisons using the Tukey were carried out. There was a significant difference between moderate and low intensities $(p=0.001)$ with sprinters on moderate intensity had the HSP70 levels on average $18.799 \mathrm{pq} / \mathrm{ml}$ more than those on low intensity. There was also a significant difference between high and low intensities $(p=0.025)$ with sprinters on high intensity had the HSP70 levels on average 12.289 $\mathrm{pq} / \mathrm{ml}$ more than those on low intensity. However, there was no significant difference between high and moderate intensities $(\mathrm{p}=0.253)$.

The principal finding of the present study was that endurance exercise led to an elevation in the resting level of peripheral leukocytes HSP70 content,as was previously reported in skeletal [8] and myocardial [9] muscle [10]. Previous studies indicated that endurance exercise elevated the resting level of HSP70 in skeletal muscle and heart [1012]. The elevation of body temperature [13, 5, 14], formation of $\operatorname{ROS}[14,15]$ and depletion of glycogen [16] are all regarded as factors that induce HSP70 expression in skeletal muscle during exercise [17]. When body temperature was maintained during endurance exercise, HSP accumulated in peripheral leukocytes. Therefore, an elevation in body temperature could be excluded as a contributor to the increase of peripheral leukocytes HSP70 in subjects. So the there is a possibility that IT with hight intensity and moderate intensity might be a stressor to induce HSP70. These findings suggest the possibility that the IT, as used in the present study, may stimulate stress response to induce HSP70.

Exercise intensity per unit time was probably higher in our exercise endurance [17]. Some of studies demonstrated that HSP70 was delayed several hours after stress: $2 \mathrm{~h}$ for heat [18, 19], $1 \mathrm{~h}$ for cold [16], $2 \mathrm{~h}$ for oxidative reagent or ethanol [20] and $2 \mathrm{~h}$ for treadmill running $[21,17]$. Endurance exercise leads to an elevation in the resting level of peripheral leukocytes HSP70. Thus it is concluded that endurance exercise elevates the resting level of peripheral leukocytes HSP70 and that the resultant accumulation of HSP70 helps to protect stress-loaded cells from injury due to the elevation of chaperone activity [17]. HSP70 production was higher in high- and moderateintensity training compared to low-intensity group in junior sprinters and these differences were statistically significant. High-intensity IT increased the concentrations of both HIF$1 \alpha$ and HSP70. This is beneficial to athletes that they may adapt to stress or hypoxic state. Hypoxia inducible factor (HIF) is a protein composed of two sub-units ( $\alpha$ and $\beta$ ) and works in hypoxia to maintain energy supply. Subunit $\alpha$ (HIF-1 $\alpha$ ) is a transcription factor which is regulated under hypoxia, while subunit $\beta$ is a constitutive protein. HIF- $1 \alpha$ concentration reflects hypoxic condition in low-, moderate-, and high-intensity IT. HIF-1 $\alpha$ is involved in angiogenesis by inducing the expression of vascular endothelial growth factor (VEGF) to maintain oxygen supply and homeostasis $[15,16,17,18,19]$. Semenza et al. [14] reported that in hypoxia, erythropoietin transcription depended on enhanced HIF-1 stability and its binding to erythropoietin gene. This cellular adaptation improved oxygen transport, induced VEGF and activated glycolytic enzymes for anaerobic glucose oxidation [14]. The authors stated that VEGF and
HIF-1 $\alpha$ did neither change after training in normoxic condition nor after low-intensity training in hypoxia, suggesting that both parameters were dependent on the intensity of training, mainly on the high-intensity, either in hypoxic or normoxic states [14].

Vogh et al. [20] reported that in normoxic and hypoxic high-intensity training, the production of HSP70 increased, whereas in normoxic low-intensity training, it decreased. During IT, the body responded to the executed training, in this case a $10 \times 100 \mathrm{~m}$ run, based on the exercise intensity. High- and moderate-intensity IT increased the athletes' physiological responses during training. These changes were seen on the increment of HIF-1 $\alpha$ and HSP70 concentrations. Moreover, the athlete sprinters were more responsive in high- and moderate-intensity IT than the controls. However, after 30-minute break, those concentrations did not fall to their normal values. This showed that the response was not easily reversed back even after the recovery period. It is suggested that more than 30 minutes are needed for an athlete to rest, especially for the one doing high-intensity IT. Besides, a shift to moderate- or low-intensity training on the following day may be needed, so that the cells have enough time to regenerate.

\section{CONCLUSION}

Concentration of HIF-1 $\alpha$ increased in all intensities of IT for sprinter-athletes, especially in the high-intensity group. After a 30-minute break, HIF-1 $\alpha$ concentration did not decrease, whereas HSP70 concentration decreased significantly after recovery in all intensity groups (although not back to baseline). Our results suggest that athletes may need more than 30-minute recovery and a shift to moderateor low-intensity training on the following day to recover after a very intense training session.

\section{ACKNOWLEDGMENT}

The author would like to thank UNY for funding this group's research.

\section{REFERENCES}

[1] Bompa T. Theory and methodology of training: the key to athletic. Dubuque: Kendall hunt publishing company; 1997

[2] Fox EL, Bowers RW, Foss LM. The physiological basis for exercise and sport. New York: Mc Graw Hill; 1993.

[3] Pate RR, McClenaghan B, Rotella R. Scientific foundations of coaching. Philadelphia: Saunders College Publishing; 1984.

[4] Brooks GA, Mercier J. Balance of carbohydrate and lipid utilization during exercise: the "crossover" concept. J Appl Physiol. 1994; 76(6): 2253-2261.

[5] Mc Carthy DA, Dale MM. The leucocytes of exercise. Sports Med. 1988; 6(6): 333-363.

[6] Nemet D, Mills PJ, Cooper DM. Effect of intense wrestling exercise on leucocytes and adhesion molecules in adolescent boys. Br J Sports Med. 2004; 38(2): 154-158.

[7] Birch K, McLaren D, George K. Sport and exercise physiology. Abington: BIOS Scientific Publishers; 2005.

[8] Prior BM, Yang HT, Terjung RL. What makes vessel grow with exercise training?. J Appl Physiol. 2005; 97(3): 1119-1128.

[9] Ohno H, Shirato K, Sakurai T, Ogasawara J, Sumitani Y, Sato S, Imaizumi K, Ishida H, Kizaki T. Effect of exercise on HIF-1 and VEGF signaling. J Phys Fit Sports Med. 2012; 1(1); 5 - 16. 
hematopoietic stem cell quiescence in vivo and accelerates blood recovery following severe irradiation. Blood. 2013; 121(5):759-769.

[18] Semenza GL. Oxygen sensing, hypoxia-inducible factors, and disease pathophysiology. Annu Rev Pathol. 2014; 9:47-71.

[19] Haase VH. Hypoxic regulation of erythropoiesis and iron metabolism. Am J Physiol Renal Physiol. 2010; 299(1):F1-F13.

[20] Vogt M, Puntschart A, Geiser J, Zuleger C, Billeter R, Hoppeler H. Molecular adaptations in human skeletal muscle to endurance training under simulated hypoxic conditions. J Appl Physiol. 2001; 91(1):173-82.

[21] Sodique NO, Enyikwola O, Ekanem AU. Exercise-induced leucocytosis in some healthy adult Nigerians. Afr J Biomed Res. 2000; 3(2):85-88.

[22] Fehrenbach, E., F. Passek, A.M. Niess, H. Pohla,C. Weinstock, H.H. Dickhuth and H.S.P. Northoff Hinnak, 2000. Expression in human leukocytes is modulated by endurance exercise, Medicine and Science in Sports and Exercise, Med. Sci. Sports Exerc., 32(3): 592600 .

[23] Gonzalez, B., R. Hernando and R. Manso, 2000. Stress proteins of 70 $\mathrm{kDa}$ in chronically exercised skeletal muscle. Pflügers Arch., 440: 42-49. 9. Powers, S.K., H.A. Demirel, H.K. Vincent, J.S. Coombes, H. Naito, K.L. Hamilton, R.A. Shanely and J. Jessup, 1998. Exercise training improves myocardial tolerance to in vivo ischemiareperfusion in the rat. Am. J. Physiol. Regul. Integr. Comp. Physiol., 275: R1468-R1477.

[24] Samelman, T.R., 2000. Heat shock protein expression is increased in cardiac and skeletal muscles of Fischer 344 rats after endurance training. Exp. Physiol., 85: 92-102. 